\title{
Ultra-strong magnesium alloy with novel nanostructures
}

\author{
WU Yuan, ZHANG Fei \& LU ZhaoPing* \\ State Key Laboratory for Advanced Metals and Materials, University of Science and Technology Beijing, Beijing 100083, China
}

Received June 14, 2017; accepted August 16, 2017; published online September 28, 2017

Citation: Wu Y, Zhang F, Lu Z P. Ultra-strong magnesium alloy with novel nanostructures. Sci China Tech Sci, 2017, 60: 1769-1770, doi: 10.1007/s11431-0179126-3

Generally, two approaches were employed to develop stronger materials: one is to increase dislocations densities or/and impede their motions, such as solid solution strengthening, grain refinement strengthening and precipitate strengthening; the other is amorphization which completely eliminates dislocations, and the deformation mechanism also changes from a dislocation-activated mode to the activation of shear bands. However, too much defects can cause a defect-softening behavior. For example, when the grain size of crystalline materials is less than $10 \mathrm{~nm}$, plastic deformation becomes dominated by grain boundary sliding and grain boundary softening, causing the reverse Hall-Petch effect [1]. The amorphous structure usually shows a restricted strain of about $2 \%$ due to the shear-band softening effect [2]. Hence, how to fabricate materials with the strength that approached their theoretically predicted value is a difficult yet important challenge.

Recently, Lu's reasearch team at City University of Hong Kong has made a breakthrough in this regard by successfully developing a dual-phase magnesium thin film with near-ideal strength [3]. They used a special magnetron-sputtering technology to fabricate a $10-\mu \mathrm{m}$-thick film whose strength reaches 3.3 gigapascals-making it the strongest magnesium alloy thin film yet achieved.

This amorphous/nanocrystalline dual-phase structure was named by the research group as supra-nano-dual-phase glass-crystal (SNDP-GC). The supra-nano means that the

\footnotetext{
*Corresponding author (email: luzp@ustb.edu.cn)
}

size of each phase is less than $10 \mathrm{~nm}$. The sputtered film has a three-dimensional homogeneous structure comprising $\mathrm{MgCu}_{2}$ grains of around $6 \mathrm{~nm}$ in diameter uniformly embedded in magnesium-enriched amorphous shells around $2 \mathrm{~nm}$ thick. The mechanical properties obtained through tests on the micrometer-sized pillars which extracted from a large piece of film are outstanding. The hardness of these pillars reaches $6.5 \mathrm{GPa}$, greater than that of most magnesium-based metallic thin films ever known. The Young's moduli of magnesium-based SNDP-GC is $65 \mathrm{GPa}$. Strength of this ultra-strong magnesium alloy has reached the proposed ideal strength of metallic glasses (i.e., E/20, E is Young's moduli). Moreover, the strength of any sub-micrometer-sized pillars in this SNDP-GC is almost identical, which means the strength of the material is not size-dependent.

The new advanced magnesium-based SNDP-GC is 10 times stronger than conventional crystalline magnesium alloys and has a super deformation capacity that is twice as high as magnesium-based metallic glasses. The strength results from the quasi-dislocation-free nanocrystals and the fully relaxed glass shells. The authors employed molecular-dynamics simulations, HRTEM and constitutive model calculation to reveal the relevant mechanisms and found that the traditional grain boundary sliding and softening mechanism were replaced by the generation of multiple embryonic shear bands in the amorphous shell. In addition, the smaller nanocrystalline cores contribute to the higher strength. Finally, the initial arrest of shear bands by the nanocrystalline $\mathrm{MgCu}_{2}$ and the strain-induced hardening of the nanocrystals promote the near-ideal strength. 
Low-density magnesium alloys are a promising type of materials for making lighter vehicles and improving fuel efficiency. One major challenge for light-weight strategy is to develop high-strength magnesium alloys. However, traditional hardening methodologies that have led to major improvements in strength of other metals have been less effective for magnesium alloys. Up to date, the most effective precipitation treatments applied to magnesium alloys have barely doubled the alloys' strength. A major obstacle to further improvement lies in the difficulty of making a uniform dispersion of closely spaced, fine precipitates. Compared to the ex-situ strengthening method, Lu's in-situ approach incorpo- rated the complementary advantages of metallic glasses and nanocrystals, giving rise to a novel three-dimensional homogeneous structure near the ideal strength. The strategy for creating this type of nanocrystalline and amorphous phase combined synthetically and efficiently, opening a different pathway to creating high-strength metals.

1 Meyers M A, Mishra A, Benson D J. Mechanical properties of nanocrystalline materials. Prog Mater Sci, 2006, 51: 427-556

2 Schuh C, Hufnagel T, Ramamurty U. Mechanical behavior of amorphous alloys. Acta Mater, 2007, 55: 4067-4109

$3 \mathrm{Wu} \mathrm{G}$, Chan K C, Zhu L, et al. Dual-phase nanostructuring as a route to high-strength magnesium alloys. Nature, 2017, 545: 80-83 